\title{
Exposure to Leishmania spp. infection and Lutzomyia spp. in individuals living in an area endemic for visceral leishmaniasis in Brazil
}

\author{
Karina Yukie Hirata[1],[2], Edenilson Borges de Oliveira Sobrinho ${ }^{[1],}$ \\ Lais Rigon ${ }^{[1]}$, Yuri Tani Utsunomiya ${ }^{[3]}$, Thaise Yumie Tomokane ${ }^{[4]}$, \\ Márcia Dalastra Laurenti ${ }^{[4]}$ and Mary Marcondes ${ }^{[5]}$
}

\author{
[1]. Universidade Estadual Paulista, Faculdade de Medicina Veterinária de Araçatuba, \\ Programa de Pós-Graduação em Ciência Animal, Araçatuba, SP, Brasil. \\ [2]. Universidade Estadual do Norte do Paraná, Faculdade de Medicina Veterinária, \\ Centro de Ciências Agrárias, Bandeirantes, PR, Brasil. \\ [3]. Universidade Estadual Paulista, Faculdade de Medicina Veterinária de Araçatuba, \\ Departamento de Apoio, Produção e Saúde Animal, Araçatuba, SP, Brasil. \\ [4]. Universidade de São Paulo, Faculdade de Medicina, Departamento de Patologia, São Paulo, SP, Brasil. \\ [5]. Universidade Estadual Paulista, Faculdade de Medicina Veterinária de Araçatuba, \\ Departamento de Clínica, Cirurgia e Reprodução Animal, Araçatuba, SP, Brasil.
}

\begin{abstract}
Introduction: This study aimed to investigate human exposure to Leishmania spp. infection and sandflies in an area endemic for the disease. Methods: The presence of antibodies specific for Leishmania spp. and saliva of Lutzomyia spp. and that of L. infantum DNA in blood were evaluated. Results: Antibodies against Leishmania spp. and sandfly saliva were observed in $20.8 \%$ and $37.7 \%$ of individuals, respectively. DNA of Leishmania spp. was amplified from the blood of one patient. Conclusions: The results suggest that Leishmania spp. infection may be underdiagnosed in this area.
\end{abstract}

Keywords: Antibodies. Humoral immunity. Sandfly saliva. Serology.

The accurate diagnosis of human visceral leishmaniasis (VL) is still challenging. VL should always be clinically suspected when a patient living in an area in which the disease is endemic presents with fever and splenomegaly, with or without hepatomegaly, and the diagnosis should be made as accurately and early as possible. However, failures in surveillance with unidentified asymptomatic cases and patients who die without confirmation of the disease contribute to the underdiagnosis of human VL in Brazil'.

Sandfly saliva contains several molecules that can modulate the host immune response and influence the course of Leishmania spp. infection, including maxadilan, which has

Corresponding author: Dra. Karina Yukie Hirata.

e-mail: kayukie@gmail.com

Orcid: 0000-0003-0136-3471

Received 26 June 2019

Accepted 30 August 2019 vasodilatory, anticoagulant, and immunosuppressive properties ${ }^{2}$. However, some evidence suggests that previous establishment of a specific immune response against sandfly saliva may reduce the infectivity of the pathogen and stimulate the development of a protective cellular immune response ${ }^{2}$. The importance of sandfly saliva in Leishmania spp. infection in humans has not yet been fully elucidated; however, the production of antibodies against the saliva has been evaluated in conjunction with the induction of delayed type hypersensitivity (DTH) in individuals infected with $L$. infantum to verify the role of such antibodies in the host immune response ${ }^{3}$.

Due to the territorial expansion of human VL in Brazil and because the disease may be underdiagnosed in individuals living in endemic areas, the present study aimed to investigate exposure to Leishmania spp. infection and sandflies in individuals who were referred to a hospital located in an area endemic for the disease. 
This study was approved by the Ethics Committee for Experimentation Involving Human Beings of São Paulo State University, Araçatuba (protocol CAEE: 39096314.8.0000.5420). The samples were obtained from individuals who were referred to a hospital in the micro-region of Araçatuba, composed of 16 counties, in São Paulo State, Brazil, an area with intense transmission of VL and high prevalence of canine visceral leishmaniasis (CanVL). Patients who needed to undergo blood collection were invited to participate in the study. The blood aliquots were separated as follows: one for the serological tests and the other for Leishmania polymerase chain reaction (PCR). Individuals were eligible for the study if (a) they were aged at least 2 years; (b) they had no previous history of VL; and (c) they lived in one of the municipalities of the micro-region. Of 1,238 individuals referred to the public hospital who underwent blood collection, 284 agreed to participate in the study.

Enzyme-linked immunosorbent assay (ELISA) for Leishmania spp. using crude L. infantum antigen (MHOM/ $\mathrm{BR} / 72 /$ strain46) and anti-human $\operatorname{IgG}$ peroxidase conjugate (Sigma-Aldrich, A6029) was performed according to the method of Laurenti et al. ${ }^{4}$. ELISA for Lu. longipalpis saliva, using as antigen salivary gland lysate (SGL) from Lu. longipalpis captured in Cametá municipality, Pará state, Brazil, was performed according to the method of Rohousova et al. ${ }^{5}$. SGL was produced according to the method of Batista et al. ${ }^{6}$. All samples were evaluated in triplicate. Negative and positive controls were included in each plate, and values were expressed as triplicate optical densities (ODs). Cutoff values were determined by analysis of serum samples from healthy individuals from an area non-endemic for VL. The mean value plus 3 standard deviations was considered as the cutoff point. The ODs were expressed in ELISA units (EUs). The cutoff points for anti-Leishmania spp. and anti-saliva were $38.51 \mathrm{EUs}$ and 29.43 EUs, respectively.

Samples of whole blood were also used for Leishmania spp. DNA amplification by PCR, according to the method of Marcondes et al. ${ }^{7}$. The target Leishmania DNA for PCR amplification was a 116-base-pair fragment in the constant region of the kinetoplast DNA minicircle. Briefly, the reaction was performed using a commercial mastermix with SYBR
Green fluorophore (SYBRGreen JumpStart TaqReadMix S4438, Sigma-Aldrich, St Louis, MO, USA), $900 \mathrm{nM}$ of each primer (JW11 (forward), 5'-CCTATTTTACACCAACCCCCAGT-3', and JW12 (reverse), 5'-GGGTAGGGGCGTTCTGCGAAA-3'), and $5 \mu \mathrm{L}$ of DNA, in a final volume of $25 \mu \mathrm{L}$. Samples (tested in triplicate) were placed into 96-well PCR plates, and PCR amplification was performed in a thermocycler (CFX96TM Real-Time System, Bio-Rad, Hercules, CA, USA) using the following conditions: $94^{\circ} \mathrm{C}$ for $2 \mathrm{~min}$ and 40 cycles of $94^{\circ} \mathrm{C}$ for $15 \mathrm{~s}$, followed by $60^{\circ} \mathrm{C}$ for $1 \mathrm{~min}$, when fluorescence data were collected. To conduct a melting curve analysis, the temperature was increased from $60^{\circ} \mathrm{C}$ to $95^{\circ} \mathrm{C}$, with an increment of $0.5^{\circ} \mathrm{C}$ every $5 \mathrm{~s}$. Each amplification run contained a positive control (DNA extracted from $1.6 \times 10^{4} \mathrm{~L}$. infantum promastigotes) in triplicate to test the proper conditions of the reagents and negative controls with ultrapure water in triplicate to monitor cross-contamination $^{8}$.

Associations between serological results and the variables, age and sex, were evaluated using Pearson's chi-squared test for statistical independence with Yates's correction for continuity. The significance level was adjusted for multiple testing using the Bonferroni correction, which resulted in a probability of $1 \%$ of wrongly rejecting the null hypothesis of no association. Additionally, the linear correlation between titers of antibodies specific for Leishmania spp. and Lutzomyia spp. was analyzed using Pearson's product-moment correlation coefficient. All statistical analyses were performed using $\mathrm{R}$ software version 3.4.3 (R Core Team, 2018).

Based on the serology and PCR results, of 284 evaluated patients, $60(21.1 \%)$ were considered to have been exposed to Leishmania spp. Data related to sex and age of the population are shown in Table 1. Antibodies against Leishmania spp. were observed in 59 (20.8\%) patients, while antibodies against sandfly saliva were observed in 107 (37.7\%). A total of 58 (20.4\%) patients were seropositive in both tests, $49(17.3 \%)$ had antisaliva antibodies without the presence of anti-Leishmania spp. $\mathrm{IgG}$, and one $(0.4 \%)$ had anti-Leishmania spp. IgG without the presence of antibodies against the sandfly saliva. In 176 $(61.9 \%)$ individuals, a negative result was observed for both serological tests.

TABLE 1: Number and percentage, according to sex and age, of 284 patients who were referred to a public hospital in an area endemic for visceral leishmaniasis, in the micro-region of Araçatuba, SP, considered exposed to Leishmania spp. according to the presence of anti-Leishmania spp. antibodies or amplification of Leishmania spp. DNA fragment by real-time PCR.

\begin{tabular}{|c|c|c|}
\hline Sex & $\begin{array}{l}\text { Total group } \\
\quad(n=284)\end{array}$ & $\begin{array}{l}\text { Exposed group } \\
\qquad(n=60)\end{array}$ \\
\hline Male & $92(32.4 \%)$ & $12(20.0 \%)$ \\
\hline Female & $192(67.6 \%)$ & $48(80.0 \%)$ \\
\hline \multicolumn{3}{|l|}{ Age group } \\
\hline $19-65$ years & $226(79.6 \%)$ & $51(85.5 \%)$ \\
\hline$>65$ years & 49 (17.3\%) & $8(13.3 \%)$ \\
\hline
\end{tabular}


The glyceraldehyde-3-phosphate dehydrogenase (GAPDH) housekeeper gene was consistently amplified from all samples subjected to PCR. Leishmania spp. DNA was amplified from the blood sample in only one patient $(0.35 \%)$, a man with nonVL-compatible clinical signs, whose serologies were negative for both Leishmania spp. and sandfly saliva. Of the patients with positive serological tests, $35.6 \%$ (21/59) had anti-Leishmania spp. EUs higher than twice the cutoff point, while $73.8 \%$ (79/107) had anti-saliva EUs higher than twice the cutoff point, reaching values of up to five times higher.

A significant association was observed between the presence of anti-Leishmania spp. and anti-saliva antibodies $(\mathrm{p}<0.01)$ (Table 2). Regarding anti-Leishmania spp. and anti-saliva EUs, a highly significant regression was observed $(\mathrm{p}<0.05)$ (Figure 1), suggesting moderate to strong correlation ( $\mathrm{r}=0.683$ ). There was no significant association between the presence of anti-Leishmania spp. antibodies and sex $(\mathrm{p}=1.000)$ or age $(\mathrm{p}=0.290)$ and between the presence of anti-saliva antibodies and sex $(\mathrm{p}=0.826)$ or age $(\mathrm{p}=0.621)$ (Table 2$)$.

The prevalence of anti-Leishmania spp. antibodies (20.8\%) in the studied population was similar to that observed in a study conducted in the state of Rio Grande do Norte (24.6\%), an area also endemic for VL in the Northeast of Brazil ${ }^{9}$. A positive serological test in humans may be used to confirm a recent or acute infection by $L$. infantum, regardless of the presence of symptoms, but does not indicate a risk of progression to the clinical form of the disease ${ }^{10}$. Generally, most individuals infected with $L$. infantum have asymptomatic infection ${ }^{10}$. In these cases, there is an increase in the serum antibody titer after the infection, followed by a decrease over time, while a predominantly cellular immune response develops, which can be evidenced by the presence of a DTH reaction. In contrast, individuals who progress to the clinical form of the disease have high titers of antibodies, which tend to decrease only after effective treatment $t^{9,10}$.

The prevalence of parasitemia identified by PCR in the studied population was $0.35 \%$, lower than the $17 \%$ observed in individuals living in neighborhoods adjacent to those with confirmed cases of VL. ${ }^{9}$ DNA amplification from the peripheral blood should always be interpreted together with the clinical presentation and results of other diagnostic tests ${ }^{11}$ and does not necessarily indicate a risk of progression of the infection. The presence of parasite DNA in the absence of serum antibodies against the parasite most likely indicates a recent infection, in which there has been insufficient time for seroconversion?.

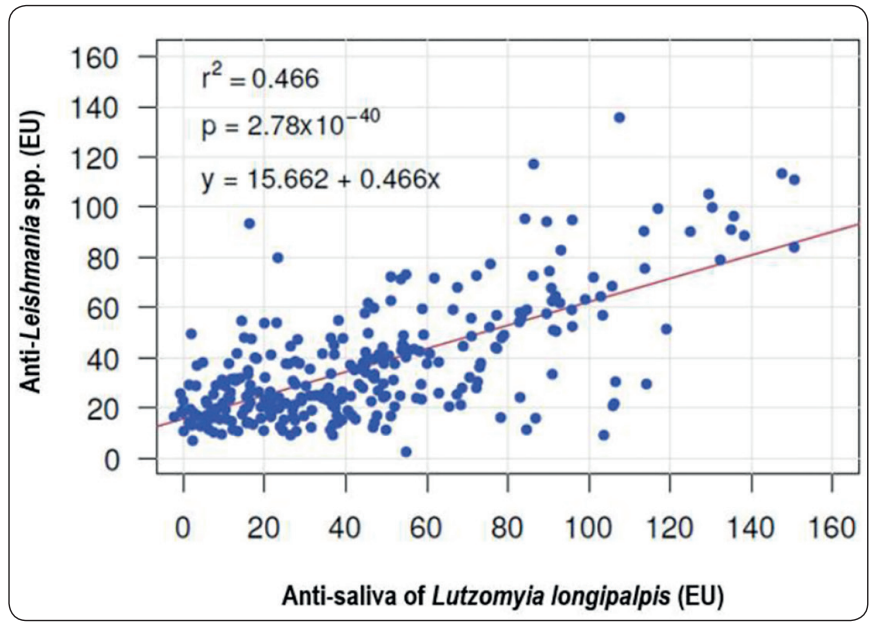

FIGURE 1: Scatter plot and adjusted regression line for anti-Leishmania spp. and anti-Lutzomyia spp. saliva antibodies (ELISA units [EUs]) from patients who were referred to a hospital in an area endemic for visceral leishmaniasis in Brazil.

About $37.7 \%$ of the evaluated patients had antibodies against sandfly saliva. The high titers of such antibody, detected in the majority of seropositive patients, could suggest that repeated exposures to the vector occur in individuals living in endemic areas $^{12}$. In the present study, $20.4 \%$ of patients had antibodies against both sandfly saliva and Leishmania spp., and there was a moderate to strong correlation between the intensities of both serological responses, varying from the result of a previous study in which children who had anti-L. chagasi antibodies did not develop a humoral immune response against the saliva of the vectors ${ }^{3}$. In the abovementioned study, individuals who did not have an immune response against salivary proteins developed anti-Leishmania antibodies associated with disease progression ${ }^{3}$. The presence of Leishmania spp. antibodies without the concomitant presence of antibodies against sandfly saliva was observed in only one $(0.4 \%)$ patient of the present study; however, we did not have access to the information on his clinical evolution.

In $17.3 \%$ of patients, it was possible to identify anti-saliva antibodies without anti-Leishmania spp. antibodies. This could be due to several factors including the possibility of being exposed to non-infected sandflies, time necessary for seroconversion after exposure to the parasite, or development of a predominantly cellular immune response in individuals who, even though infected, did not have detectable antibodies

TABLE 2: Associations between the presence of anti-Leishmania spp. and anti-saliva of Lutzomyia spp. antibodies, sex and age of 284 patients who were referred to a public hospital in an area endemic for visceral leishmaniasis, in the micro-region of Araçatuba, SP.

\begin{tabular}{ccccc}
\hline Response variable & Independent variable & Statistic $\left(\chi^{2}\right)^{\mathbf{a}}$ & Degrees of freedom & P-value \\
\hline Anti-Leishmania & Sex & 0.000 & 1 & 1.000 \\
Anti-Leishmania & Age & 2.473 & 2 & 0.290 \\
Anti-Leishmania & Anti-saliva & 113.345 & 1 & $p<0.01$ \\
Anti-saliva & Sex & 0.048 & 1 & 0.826 \\
Anti-saliva & Age & 0.954 & 2 & 0.621 \\
\hline
\end{tabular}

aPearson's test of independence. 
against Leishmania spp. ${ }^{9,10}$. Sandfly saliva contains several pharmacologically active substances capable of interfering with the host's inflammatory and immunological responses. In naive hosts, co-inoculation of sandfly saliva and parasites increases the likelihood of infection ${ }^{13}$; however, hosts that are repeatedly exposed to sandflies and exhibit high titers of anti-saliva antibodies develop a protective immune response against Leishmania spp. infection ${ }^{13}$. This suggests that significant exposure to the bites of sandflies could be associated with the development of a protective immune response against Leishmania, driven by factors contained within the vector saliva ${ }^{14}$.

The stimulation of a cellular immune response against the parasite after previous contact with $\mathrm{Lu}$. longipalpis saliva may be due to a DTH reaction at the site of the bite, which may transform the lesion and its surroundings into an inhospitable site for the development of Leishmania infection ${ }^{12}$.

Although several studies have been conducted with the same technique, using a crude $L u$. longipalpis SGL to obtain the antigen ${ }^{2,3}$, it is impossible to rule out a cross-reaction between the antigens present in the SGL and those present in the saliva of other insects ${ }^{2}$. Moreover, some points regarding the limitation of serological tests should be considered, such as false negative results in cases of low antibody titers, reflecting the sensitivity of the test, and false positive results in the presence of co-infections, reflecting the specificity of the test. Another point that should be considered is that asymptomatic individuals, such as those evaluated in this study, generally present low circulating antibody titers, due to either low and/ or recent exposure or the predominance of a cellular immune response over humoral immune response ${ }^{10}$, compromising the accuracy of the serological test ${ }^{15}$.

It is important to highlight that approximately $20 \%$ of patients living in an area endemic for VL, who were referred to a hospital with a diagnosis of fever of unknown origin, had anti-Leishmania spp. antibodies, but none of them had $\mathrm{VL}$ as a possible diagnosis. Therefore, the results of the present study suggest that infection by Leishmania spp. may be underdiagnosed in the region. VL should be included in the differential diagnosis of patients referred to hospitals, particularly in endemic areas.

\section{ACKNOWLEDGMENTS}

We offer our deepest appreciation to the institutions that provided technical support for the development and implementation of this study.

\section{Conflict of interest}

The authors declare that they have no conflict of interest.

\section{Financial Support}

This work was supported by the Coordination of Superior Level Staff Improvement (CAPES), PhD Scholarship, and São Paulo Research Foundation (FAPESP) - Scientific Initiation Scholarship (project 2015/02302-0).

\section{REFERENCES}

1. BRASIL. Ministério da Saúde. Leishmaniose visceral. 2017. [Updated 2018 jun 12; Cited 2018 feb 03]. Available in: http:// portalms.saude.gov.br/saude-de-a-z/leishmaniose-visceral

2. Aquino DM, Caldas AJ, Miranda JC, Silva AA, Barral-Netto M, Barral A. Epidemiological study of the association between antiLutzomyia longipalpis saliva antibodies and development of delayed-type hypersensitivity to Leishmania antigen. Am J Trop Med Hyg. 2010;83(4):825-7.

3. Gomes RB, Brodskyn C, Oliveira CI, Costa J, Miranda JC, Caldas A et al. Seroconversion against Lutzomyia longipalpis saliva concurrent with the development of anti-Leishmania chagasi delayed-type hypersensitivity. J Infect Dis. 2002;186(10):1530-4.

4. Laurenti MD, Leandro Jr MV, Tomokane TY, De Lucca HRL, Aschar M, Souza CSF, et al. Comparative evaluation of the DPP ${ }^{\circledR}$ CVL rapid test for canine serodiagnosis in area of visceral leishmaniasis. Vet Parasitol. 2014;205(3-4):444-50.

5. Rohousova I, Ozensoy S, Ozbel Y, Volf P. Detection of speciesspecific antibody response of humans and mice bitten by sand flies. Parasitology. 2005;130(5):493-9.

6. Batista LFS, Da Matta VLR, Tomokane TY, Pacheco AD, Silveira FT, Rossi CN, et al. Canine antibody response to Lutzomyia longipalpis saliva in endemic area of visceral leishmaniasis. Rev Soc Bras Med Trop. 2016;49(3):361-4.

7. Marcondes M, Hirata KY, Vides JP, Sobrinho LSV, Azevedo JS, Vieira TSWJ, et al. Infection by Mycoplasma spp., feline immunodeficiency virus and feline leukemia virus in cats from an area endemic for visceral leishmaniasis. Parasit Vectors. 2018;11(131):1-8.

8. Ranasinghe S, Rogers ME, Hamilton JG, Bates PA, Maingon RD. A real-time PCR assay to estimate Leishmania chagasi load inits natural sand fly vector Lutzomyia longipalpis. Trans R Soc Trop Med Hyg. 2008;102(9):875-82.

9. Lima ID, Queiroz JW, Lacerda HG, Queiroz PVS, Pontes NN, Barbosa JDA, et al. Leishmania infantum chagasi in Northeastern Brazil: asymptomatic infection at the urban perimeter. Am J Trop Med Hyg. 2012;86(1):99-107.

10. Silveira FT, Lainson R, Crescente JA, Souza AAA, Campos MB, Gomes $\mathrm{CMC}$, et al. A prospective study on the dynamics of the clinical and immunological evolution of human Leishmania (L.) infantum chagasi infection in the Brazilian Amazon region. Trans R Soc Trop Med Hyg. 2010;104(8):529-35.

11. Ruiter CM, Van Der Veer C, Leeflang MMG, Deborggraeve S, Lucas C, Adams ER. Molecular tools for diagnosis of visceral leishmaniasis: systematic review and meta-analysis of diagnostic test accuracy. J Clin Microbiol. 2014;52(9):3147-55.

12. Vinhas V, Andrade BB, Paes F, Bomura A, Clarencio J, Miranda JC. et al. Human anti-saliva immune response following experimental exposure to the visceral leishmaniasis vector, Lutzomyia longipalpis. Eur J Microbiol Immunol. 2007;37(11):3111-21.

13. Rohoušová I, VolfP. Sand fly saliva: effects on host immune response and Leishmania transmission. Folia Parasitol. 2006;53(3):161-71.

14. Laurenti MD, Chaves LF, Tomokane TY, Abbasi I, Warburg A, Silveira FT. Reduced Leishmania (L.) infantum chagasi parasitic loads in humans exposed to Lutzomyia longipalpis bites in the Amazon region of Brazil. Parasitol Open. 2017;3:1-10.

15. Calzada JE, Saldaña A, González K, Rigg C, Pineda V, Santamaria $\mathrm{AM}$, et al. Cutaneous Leishmaniasis in dogs: is high seroprevalence indicative of a reservoir role? Parasitol. 2015;142(9):1202-14. 